\title{
Research on Temperature Points Selection of the Non-source Temperature Calibration Method Based on Pyrometer Transfer Function
}

\author{
Sun Kun*, Wang Jing, Wang Hongchao, Yu Limei, Li Chenyang and Zhang \\ Xiaowen \\ The Higher Educational Key Laboratory for Measuring \& Control Technology \\ and Instrumentations of Heilongjiang Province, Harbin University of Science and \\ Technology, Harbin, China \\ *sunkun1982@126.com
}

\begin{abstract}
The non-source temperature calibration method of the multi-spectral pyrometer (MSP), put forward in the previous article, is the non-source calibration method based on the MSP transfer function (NCCTF). In this paper, the practicability of NCCTF is researched. The situations that maybe encountered of the temperature points in the actual calibration process are simulated. The impact of the intervals temperature points of different number and unequal intervals temperature points on the NCCTF accuracy are researched theoretically to determine the temperature points select method. Simulation experimental results show that the NCCTF can meet the actual calibration process with good usability.
\end{abstract}

Keywords: High-temperature measurement, Non-source temperature calibration, Derivative extrapolation; Temperature points selection

\section{Introduction}

Calibration technique is a key technology in the field of multispectral temperature measurement, in a sense, to study it even more important than the study of multi-spectral pyrometer instrument itself [1-3]. At present, Multi-spectral pyrometer used in hightemperature measurement has already had high resolution and high signal to noise ratio. However, the non-source temperature (higher than $3000^{\circ} \mathrm{C}$ ) calibration falls far behind the development of multi-spectral pyrometer and the existing methods which are the onepoint calibration method and the warming filter calibration method have already seriously hindered the precision and application range of the pyrometer [4-5]. In order to break through the limitation of calibration of non-source temperature, a new calibration method has been put forward in previous paper. The temperature-voltage (T-U) model has been formed based on the MSP transfer function where output voltage U of the MSP is derived from its corresponding known temperature point. Based on the model, Derivative least square method has been used to obtain the parameters of the model to realize the nonsource temperature calibration. Both theoretical and experimental data have already proved the efficiency and precision of the calibration method. In addition, in the spectral range of multispectral high temperature measurement applications $(0.4 \sim 1.1 \mu \mathrm{m})$, the influence of work wavelength on the accuracy of NCCTF has been analyzed [6-7].

On this above, the simulation experiment of the NCCTF are performed in this article under the situations that may be encountered of the temperature points selection. The influence on the NCCTF accuracy of different situations is researched to draw the temperature points selection rule. 


\section{Measurement Principle}

The actual temperature $T$ of blackbody furnace has the following relationship with output voltage $U$ when the working wavelength is $\lambda$ :

$$
\ln U=a+\frac{b}{T}+\frac{c}{T^{2}},
$$

In function (1), a, b and $\mathrm{c}$ are the unknown value, let

$$
\begin{gathered}
Y=\ln U, \\
X=\frac{1}{T},
\end{gathered}
$$

then,

$$
Y=a+b X+c X^{2},
$$

Because derivative least-square [8] not only realizes the curve fitting, but also simultaneously realizes the fitting of tendency of curve change; therefore, obtaining $a, b$ and $\mathrm{c}$ with derivative least-square will be able to control the tendency and ensure the extrapolation precision better. Then, using derivative least-square, let

$$
\begin{aligned}
& Q_{0}=\sum_{i=1}^{n}\left(Y_{i}-a-b X_{i}-c X_{i}^{2}\right)^{2}, \\
& Q_{1}=\sum_{i=1}^{n-1}\left(Y_{i}^{\prime}-b-2 c \bar{X}_{i}\right)^{2},
\end{aligned}
$$

Then, the model parameters $\mathrm{a}, \mathrm{b}$ and $\mathrm{c}$ can be obtained from the following functions.

$$
\begin{aligned}
& \frac{\partial Q_{0}}{\partial a}=-\sum_{i=1}^{n} w_{i}\left(Y_{i}-a-b X_{i}-c X_{i}^{2}\right)=0 \\
& \frac{\partial Q_{0}}{\partial b}=-\sum_{i=1}^{n} w_{i}\left(Y_{i}-a-b X_{i}-c X_{i}^{2}\right) \times X_{i}=0 \\
& \frac{\partial Q_{1}}{\partial c}=-\sum_{i=1}^{n-1} w_{i}^{\prime}\left(Y_{i}^{\prime}-b-2 c \overline{X_{i}}\right) \times 2 \overline{X_{i}}=0 \\
& c=\frac{S_{\bar{x}}\left(S_{x} S_{y}-n S_{x y}\right)-S_{\overline{x y}}\left(S_{x}^{2}-n S_{x x}\right)}{S_{x}\left(S_{x x} S_{x}-n S_{x x x}\right)-2 S_{\overline{x x}}\left(S_{x}^{2}-n S_{x x}\right)} \\
& b=\frac{S_{\overline{x y}},-2 c S_{\overline{x x}}}{S_{x_{i}}} \\
& S_{x}-b S_{x y}-c S_{x x} \\
& S_{x}=\sum_{i=1}^{n} S_{w} w_{i} X_{i}, \\
& S_{y}=\sum_{i=1}^{n} w_{i} Y_{i}, \\
& =\sum_{i=1}^{n} w_{i} X_{i} Y_{i},
\end{aligned}
$$




$$
\begin{aligned}
& S_{\bar{x} y^{\prime}}=\sum_{i=1}^{n-1} w_{i} \bar{X}_{i} Y_{i}^{\prime}, \\
& S_{x x}=\sum_{i=1}^{n} w_{i} X_{i}^{2}, \\
& S_{x x x}=\sum_{i=1}^{n} w_{i} X_{i}^{3}, \\
& S_{\overline{x x}}=\sum_{i=1}^{n-1} w_{i} \bar{X}_{i}^{2}, \\
& S_{w}=\sum_{i=1}^{n} w_{i}, \\
& Y_{i}^{\prime}=\frac{Y_{(i+1)}-Y_{i}}{X_{(i+1)}-X_{i}, i=1,2, \cdots, n-1,} \\
& \overline{X_{i}}=E_{i+1}+(1-\varepsilon) X_{i}, i=1,2, \cdots, n-1, \\
& T_{i}=\frac{-b-\sqrt{b^{2}-4 c\left(a-\ln U_{i}\right)}}{-b} .
\end{aligned}
$$

Where $w_{i}$ and $w_{i}^{\prime}$ are the corresponding weight of known temperature $T_{i}$. The rules of value-taking are 1) the weight value near extrapolation range is large; 2) the weight value of actual measurement is large. However, in the process of $100^{\circ} \mathrm{C}$-interval calibration of known temperature range, every calibration point is subjected to random and systemic errors, and the relationship of which is hard to predict. Therefore, in order to reduce the interference of the error of one point to the whole curve, let $w_{i}=w_{i}{ }_{i}$, and use the known temperature point near the extrapolation range. In function (22), parameter $\varepsilon$ is set as $1 / 2$ in actual practice. Function (23) is the temperature calculation function.

\section{The Selection of the Temperature Points}

The NCCTF with good usability requires having a good ability to adapt the situations that maybe encountered. By changing the number of the intervals selected temperature points and establishing the unequal intervals temperature points to simulate the practical 100-interval temperature calibration in source temperature, the effects on the extrapolation accuracy of the NCCTF are analyzed.

\subsection{Different Number of Intervals Temperature Points}

Theoretically, blackbody temperature-radiation curve is used to verify the method. The specific method is as follows: the $2600{ }^{\circ} \mathrm{C}$ is taken as the beginning point and $3000^{\circ} \mathrm{C}$ as the ending point. The number of $5,8,10,15,20,25$ intervals temperature points are taken respectively in this range, the Planck function calculation values of interval points are taken as calibration value of source temperature range. In the non-source temperature range from $3100{ }^{\circ} \mathrm{C}$ to $4800{ }^{\circ} \mathrm{C}$, each interval $50{ }^{\circ} \mathrm{C}$ temperature points are taken as extrapolation points. The Planck calculation value at temperature point over $3000^{\circ} \mathrm{C}$ is taken as the output of MSP. Then, through extrapolation, the corresponding temperature value is obtained. The sum of the extrapolation point error squares can be calculated. When work wavelength is $0.6 \mu \mathrm{m}$, the relation curve of extrapolation temperature range and sums of extrapolation temperature point error squares are shown in Figure1, and the relation curve in the range from $3100{ }^{\circ} \mathrm{C}$ to $3500^{\circ} \mathrm{C}$ are shown in Figure 2. When work 
wavelength is $0.9 \mu \mathrm{m}$, the relation curves are shown in Figure 3 and the relation curve in the range from $3100{ }^{\circ} \mathrm{C}$ to $3500{ }^{\circ} \mathrm{C}$ are shown in Figure 4 .

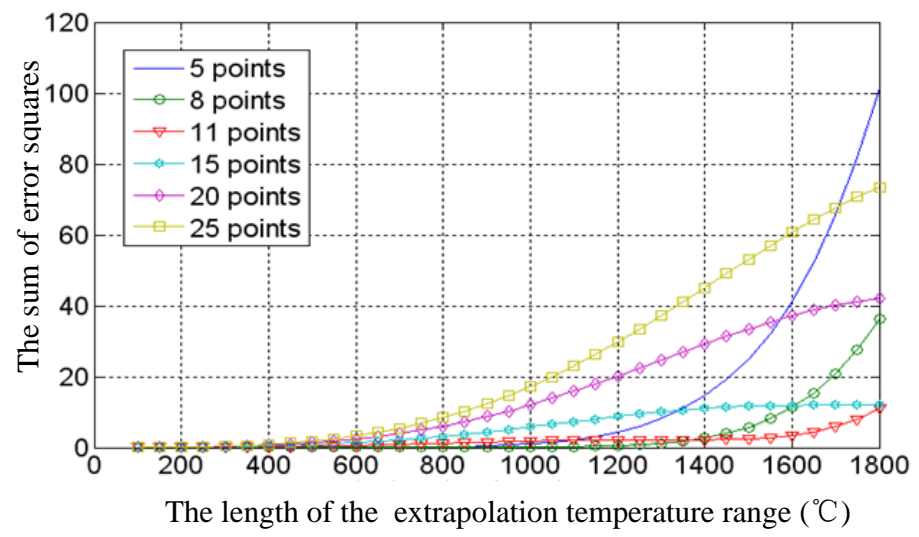

Figure 1. The Curves 2 of Extrapolation Temperature Range and Sums of Extrapolation Temperature Point Error Squares $(\lambda=0.6 \mu \mathrm{m})$

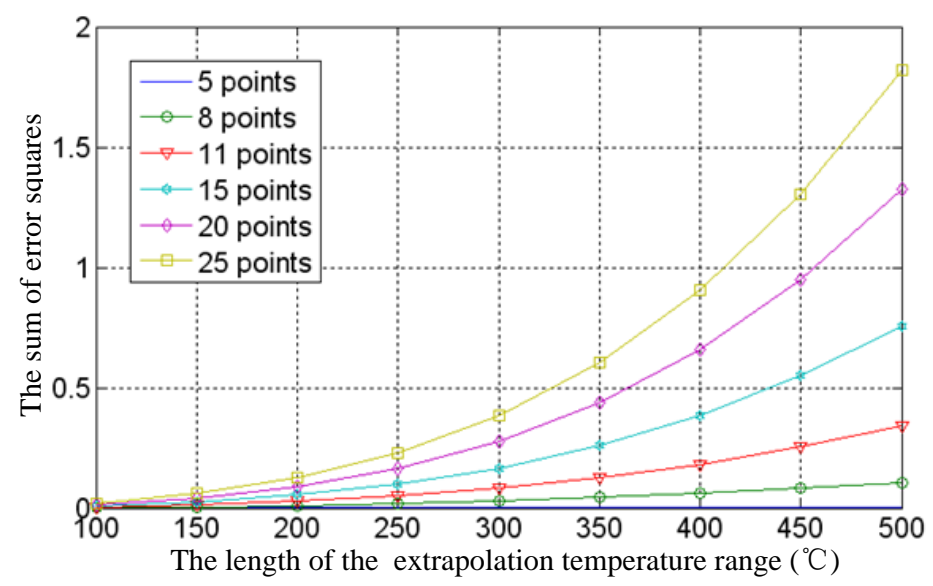

Figure 2. The Curves 2 of Extrapolation Temperature Range and Sums of Extrapolation Temperature Point Error Squares $\left({ }_{\lambda}=0.6 \mu \mathrm{m}\right)$

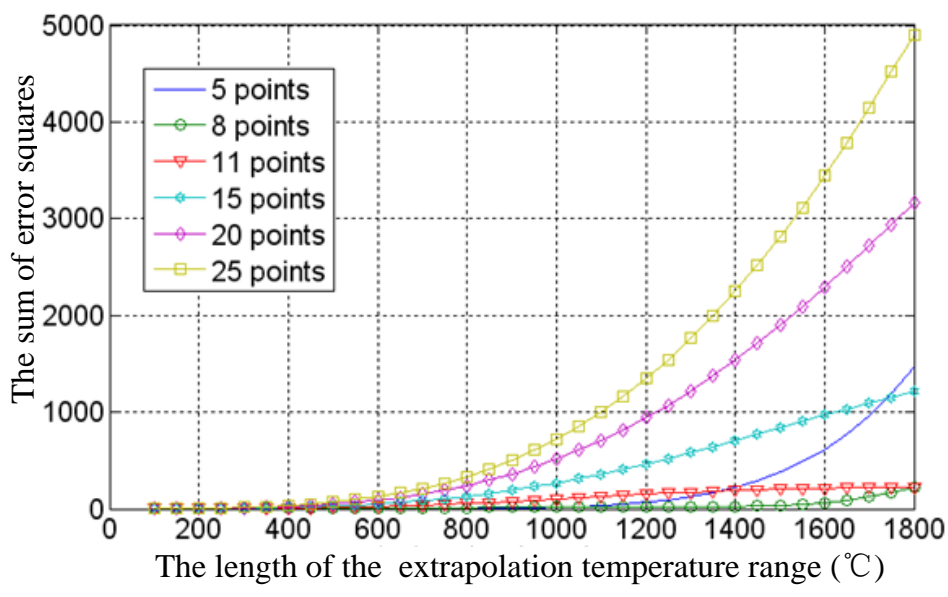

Figure 3. The Curves 1 of Extrapolation Temperature Range and Sums of Extrapolation Temperature Point Error Squares $\left({ }_{\lambda}=0.9 \mu \mathrm{m}\right)$ 


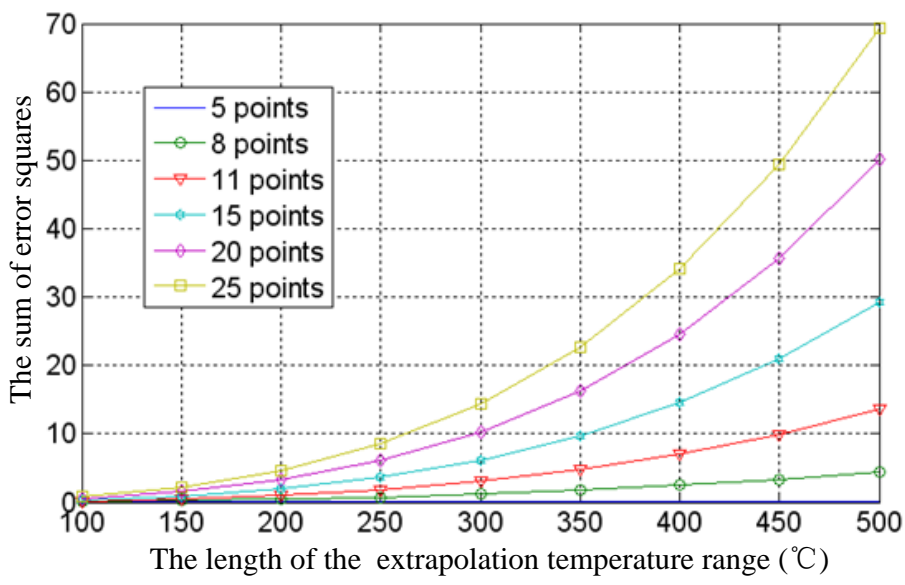

\section{Figure 4. The Curves 2 of Extrapolation Temperature Range and Sums of Extrapolation Temperature Point Error Squares $\left({ }_{\lambda}=0.9 \mu \mathrm{m}\right)$}

Conclusions can be drawn from the experiment above.

a. The number of the temperature has less effect on the accuracy of the NCCTF. Take the figure 2 for example, in the extrapolation temperature range from $3100^{\circ} \mathrm{C}$ to $3500^{\circ} \mathrm{C}$, the maximum difference of the absolute error squares sum is 2 when the number of the point is 2 .

b. The best number of the NCCTF application points related with the length of the extrapolation temperature range and wok wavelength. When the work length is $0.6 \mu \mathrm{m}$ and the length of the extrapolation temperature range is $500{ }^{\circ} \mathrm{C}$, the best number of the application points is 5 . When the work wavelength is $0.6 \mu \mathrm{m}$ and the extrapolation range is $1300^{\circ} \mathrm{C}$, the best number of the points is 8 . When the work wavelength is $0.6 \mu \mathrm{m}$ and the extrapolation range is $1800{ }^{\circ} \mathrm{C}$, the best number of the points is 11 . When the work wavelength is $0.9 \mu \mathrm{m}$ and the extrapolation range is $500^{\circ} \mathrm{C}$, the best number of the points is 5 . When the work wavelength is $0.9 \mu \mathrm{m}$ and the extrapolation range is $1800^{\circ} \mathrm{C}$, the best number of the points is 8 .

\subsection{Unequal Intervals Temperature Points}

Within the temperature range of $2600{ }^{\circ} \mathrm{C}$ and $3000{ }^{\circ} \mathrm{C}$, five temperature points that diverge $\pm 2{ }^{\circ} \mathrm{C}$ from 100 -interval temperature are selected and extrapolated with this method. The process is repeated 10000 times. In practice, the temperature points selected near $3000^{\circ} \mathrm{C}$ are within the range of $2998^{\circ} \mathrm{C}$ and $3000^{\circ} \mathrm{C}$, and the rest of the points are all within the divergence of $\pm 2^{\circ} \mathrm{C}$. Then, the curve of the maximum and minimum value of extrapolation temperature points error are shown as figure 5 . When the divergence is $\pm 4^{\circ} \mathrm{C}$, the curve is shown as Figure 6. When the divergence is $\pm 5^{\circ} \mathrm{C}$, the curve is shown as Figure 7. 


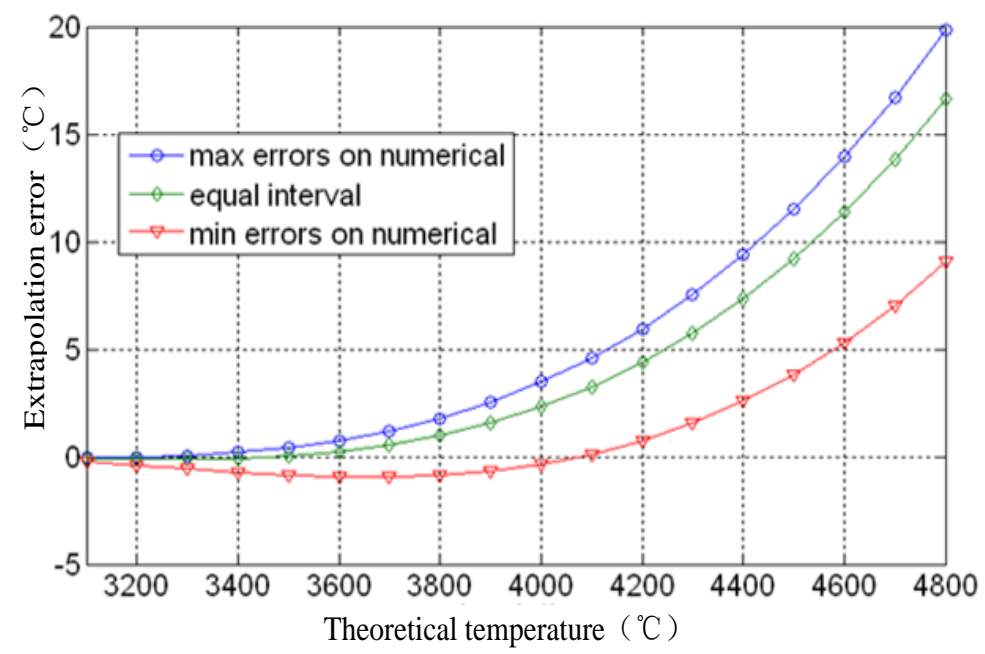

Figure 5. The Extrapolation Error Curves of Points within $\pm 2^{\circ} \mathrm{C}$ to 100 interval Temperature

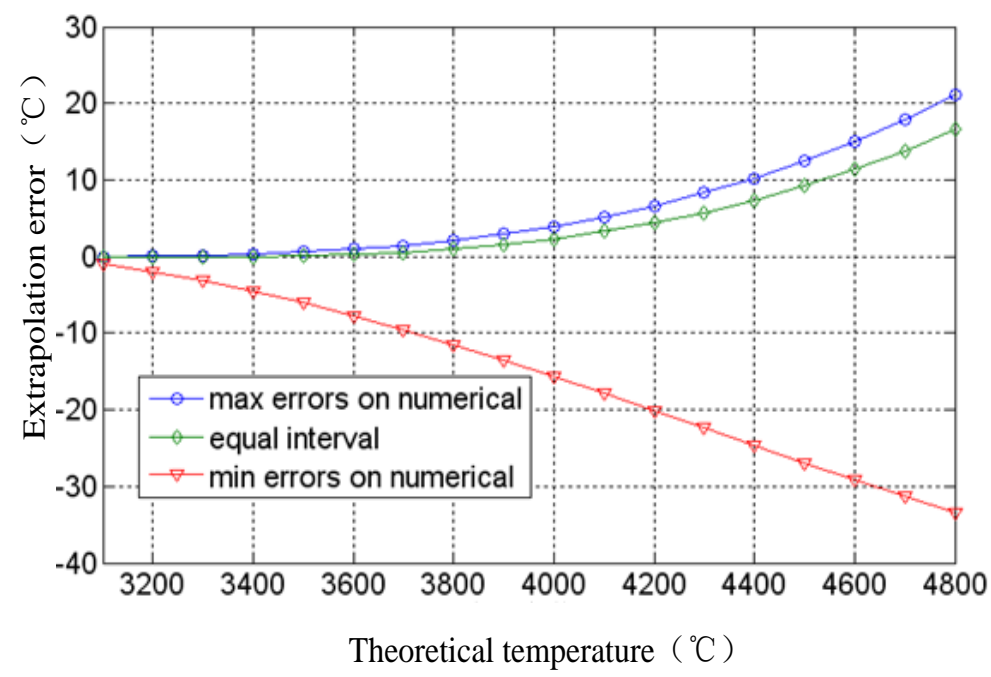

Figure 6. The Extrapolation Error Curves of Points within $\pm 4^{\circ} \mathrm{C}$ to 100 interval Temperature 


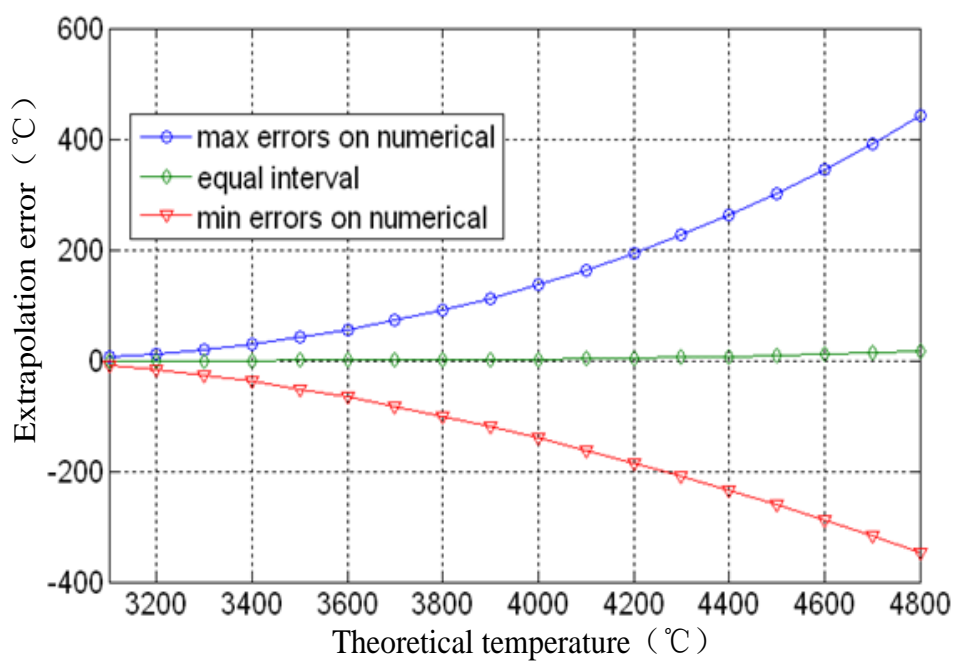

\section{Figure 7. The Extrapolation Error Curves of Points within $\pm 5^{\circ} \mathrm{C}$ to 100 - interval Temperature}

From the simulation results of the uneven interval temperature point theory, it can be known that:

When the interval between selected temperature point and the 100-interval temperature increases, the extrapolation precision uncertainty grows. Take the working wavelength of $0.9 \mu \mathrm{m}$ as an example, in order to limit the uncertainty range within the ideal precision, the temperature point interval has to be within the divergence of $\pm 4^{\circ} \mathrm{C}$. If the interval is greater than $\pm 4^{\circ} \mathrm{C}$, this method should be used to theoretically testify the system precision. The temperature points should consequently be readjusted if the precision requirement cannot be satisfied.

The same method has been used to simulate the effect of uneven interval of temperature points on the extrapolation precision for different wavelengths. The following data are obtained:

In the spectral range from $0.4 \mu \mathrm{m}$ to $1.1 \mu \mathrm{m}$, the smaller the wavelength is, the greater the interval between the temperature point and 100-interval temperature. When wavelength is smaller than $0.9 \mu \mathrm{m}$, the interval can be greater than $\pm 4^{\circ} \mathrm{C}$. When the wavelength is greater than $0.9 \mu \mathrm{m}$, in order to ensure the extrapolation calibration precision, the maximum interval is $\pm 4^{\circ} \mathrm{C}$.

Therefore, it can be seen that the method has the capability of adjustment for uneven temperature intervals.

\section{Conclusion}

In this paper, by simulating the pratical situation that temperature points may appear in the process of the calibration, the simulation experiments of the NCCTF are researched. The simulation results show that the NCCTF is able to adapt the situations that that maybe encountered in the calibration.

\section{Acknowledgments}

This research is supported by Technology Research Project of Heilongjiang Province Education Department (12541104). The constructive comments from the reviewers are of this paper gratefully acknowledged which have helped the author to improve the paper. 


\section{References}

[1] K. Sun, Spectrosc. Spectral. Anal, vol. 3, no. 31, (2011).

[2] K. Sun, Spectrosc. Spectral. Anal, vol. 6, no. 31, (2013).

[3] X.G. Sun, 2012 IMCCC, (2012) November15-18; Harbin, China.

[4] P. Coppa, G. Ruffino and A. Spena, High Temperature - High Pressure, vol. 20, (1988).

[5] H. Jin and W. H. XU, Chinese Journal of Instrument, vol. 3, no. 20, (1999).

[6] X. SUN and K. Sun, Spectral. Anal, vol. 1, no. 32, (2012).

[7] K. Sun and X.Sun, Proc. SPIE 8201O, (2011) November 25-28; Beijing, China.

[8] H. Fu and S. Zhang, J. Mech. Strength, vol. 1, no. 25, (2003). 